\title{
Un lamento del siglo XIX: crisis económica, pobreza educativa
}

\begin{abstract}
T AS carencias educativas preocuparon profundamente a 1 algunos novohispanos, tres o cuatro de los cuales, como diputados a las Cortes de Cádiz, habían participado activamente en proyectos que ansiaban poner en marcha en su suelo natal. ${ }^{1}$ Sin embargo, la debilidad del Estado mexicano, la poca solidaridad política y social y la falta de compromiso entre sus dirigentes para remediar la carencia de escuelas y maestros caracterizaron la primera mitad del siglo. Una clase burguesa naciente acumulaba riquezas sin preocuparse por crear un sistema educativo nacional. Puesto que esta clase tenía la posibilidad de educar a sus hijos en el extranjero o con preceptores particulares, la instrucción de los grupos populares le interesaba sólo en cuanto le facilitaba el control social. No se había instituido un impuesto progresivo sobre la renta ni sobre la propiedad, de modo que los pudientes no contribuian más que cualquier otro ciudadano (los notoriamente pobres estaban exentos); el Estado nacional, aún muy frágil y vacilante, carecía de capacidad extractiva frente a las clases dominantes. La naturaleza geográfica del país tampoco favorecía una distribución equitativa de recursos; las comunidades aisladas del centro y de las rutas comerciales no recibian ningún subsidio de las locálidades más ricas, de manera que se vieron en la necesidad de conseguir, allí donde difícilmente podía haber excedentes de su producción ganadera o agrícola, fondos para mantener a sus escuelas.
\end{abstract}

1 Nettie Lee Benson, ed., Mexico and the Spanish Cortes (1810-1822) Eight Essays, Austin, University of Texas Press, 1966. 
La acumulación de capital en manos de agiotistas y especuladores en bienes raíces creó un grupo pequeño y adinerado, a despecho de los conflictos políticos y del lento desarrollo económico del siglo, que hizo poco por resolver el problema educativo. Los sectores medios y sobre todo profesionales no tenían recursos económicos para solucionar estas carencias, pues por lo general sus conocimientos sobrepasaban en mucho a sus ingresos. Gran parte de la enorme población rural y agrícola, sumergida en la pobreza, la incomunicación, la ignorancia, el alcoholismo y una abierta oposición a las escuelas, debido a una desconfianza tradicional, ponía tremendos obstáculos a la escolarización.

Los "hombres pensantes" de la nación habían albergado una enorme fe en su capacidad para transformarse en una sociedad moderna. Como describe un libro de Javier Ocampo, Las ideas de un día, ${ }^{2}$ éstas florecieron en un ambiente de gran entusiasmo. México, ya libre de trabas legales españolas, se decía, encontraría su lugar entre el concierto de las naciones civilizadas como un país rico, apacible, ordenado, con ciudadanos de elevado nivel moral, educados, y sobre todo felices. El tema de la felicidad es frecuente en los folletos, discursos, proyectos y leyes de la primera República Federal. El optimismo era tal que, se pensaba, un buen gobierno podría conducir a la sociedad a su perfeccionamiento. Este anhelo se manifiesta en las constituciones como la Política de la Monarquía Española de 1812 y las de los estados de la República. Ejemplo de esta manera, quizá ingenua, de ver los hechos es la primera constitución de Veracruz que inclusive pone fecha limite para alfabetizar a los adultos, so pena de que éstos perdieran sus derechos ciudadanos. $^{3}$

La aparente cornucopia de recursos físicos y psíquicos del país inspiró visiones de futura paz y prosperidad. Las ideas de la Ilustración otorgaban una confianza muy grande al poder salvador y redentor de la educación. Creían los "hombres pensantes", como a sí mismo se llamaban, que la multiplicación

2 Javier Ocampo, Las ideas de un día; el pueblo mexicano ante la consumación de su independencia, México, El Colegio de México, 1969,376 pp.

3 1825-1975. 150 años de constitucionalismo veracruzano. Las constituciones políticas del estado de Veracruz, Xalapa, Gobierno del Estado, 1975, p. 72. 
de las escuelas haría tanto por la vida moral y política como la multiplicación bíblica de los panes habia hecho por los hambrientos. Estaban convencidos de la fórmula "más escuelas equivale a mejores ciudadanos". Estas instituciones milagrosas harían de los niños hombres respetuosos, obedientes, dispuestos a defender con la vida al gobierno republicano; serían responsables, instruidos, amantes del trabajo, buenos padres de familia, devotos católicos, reverentes ante el orden divino y terrenal.

Las escuelas ocupaban un lugar de gran importancia en el esquema de vida nacional; eran una pieza clave en la formación de un Estado moderno y en su sobrevivencia como país independiente. Al respecto estuvieron de acuerdo masones escoceses y yorkinos, políticos moderados y exaltados, simpatizantes del nuevo régimen y nostálgicos del viejo, más todos los grupos que se identificaban en primer lugar como mexicanos. ${ }^{4}$ (De este conjunto se excluyen necesariamente muchos nativos que vivían en comunidades cuyas actitudes regionales les impedian sentirse parte de la nación.) ¿ Por qué, entonces, la educación no prosperó, por qué no recibió el apoyo que su jerarquía, como prioridad nacional, parecía merecer?

Esta paradoja (urgencia de educación-abandono) puede explicarse, en gran medida, por lo que ahora resulta obvio. No se puede crear un sistema educativo con buenos deseos ni con el trabajo de voluntarios ni con maestros profesionales con sueldos de hambre. Últimamente se había dicho, a propósito de la bonanza económica que prometía el petróleo, que el dinero no todo lo resuelve. Sin embargo, se comprende que su ausencia explica en gran medida no sólo el atraso, sino también la imposibilidad de vencer la inercia, de establecer desde la nada un sistema de educación formal que llegara a cubrir la demanda nacional.

Una mirada a la situación económica de los maestros y el destino que realmente tuvieron los fondos para la instrucción pública nos dejaría vislumbrar la enseñanza tan deficiente reci-

4 Edmundo O'Gorman, México, el trauma de su historia, México, Universidad Nacional Autónoma de México, 1977. Es impresionante la medida en que grupos dominantes, pero violentamente opuestos, coinciden a lo largo del siglo, en sus metas finales. 
bida por la mayor parte de los niños mexicanos en el siglo pasado (que no parece tan escandalosa si se compara con el estado de la educación pública en Inglaterra o en el resto de Europa en la misma época). La injerencia de gobiernos centrales, federales y municipales, según el régimen político en el poder, también explicaría en alguna medida la distribución de recursos; su procedencia insegura y esporádica es otro punto para examinar la relación entre la crisis económica que duró en forma aguda desde la Independencia hasta la República Restaurada y la pobreza de esfuerzos y sobre todo de logros educativos durante el mismo tiempo.

Las escuelas públicas y privadas durante la Colonia fueron de varios tipos. Una real cédula de 1817 , que no hacía más que recoger mandatos similares anteriores, ordenaba la apertura de escuelas gratuitas en los conventos y en las parroquias, donde la Iglesia pagaba a un laico, maestro o maestra, para enseñar las primeras letras. Estaban además las escuelas del ayuntamiento (que aparecen ya a finales de la Colonia), pagadas por esta corporación civil. Lo más común, sin embargo, era mandar a los niños, y, después de los seis o siete años de edad solamente, a las niñas, a una "amiga", como se llamaban las escuelitas que las mujeres, a veces analfabetas, abrían en sus casas, y cuyo escaso sustento se lograba con las pequeñas cuotas pagadas por los padres de familia. Los niños con ciertas posibilidades económicas recibían su instrucción primaria en los colegios menores de las órdenes religiosas, donde pagaban colegiaturas o recibian becas, según el caso. Otras escuelas, que existían durante la colonia y fueron comunes durante el siglo XIX, fueron las que hoy llamariamos "particulares", establecidas como un negocio o servicio particular, sin intervención del gobierno. La diferencia entre pública y particular no tenía relación con la paga de colegiaturas. Había escuelas públicas, donde se tenía que contribuir con alguna cuota, y particulares, donde los alumnos pobres asistían gratuitamente, pero que eran costeadas por padres de familia con suficiente dinero o por organizaciones como la Compañía Lancasteriana ${ }^{5}$ o el Colegio de las Vizcainas.

\footnotetext{
${ }^{5}$ Un detallado estudio sobre el tema se encuentra en Dorothy Tanck de Estra-
} 
Hubo dos clases de participación estatal durante el primer medio siglo de vida independiente. Bajo el federalismo, el municipio ejercía jurisdicción sobre la instrucción primaria; el gobierno nacional no intervenía, ya que cada estado era libre y soberano. Los municipios se enfrentaban a una tarea especialmente difícil, y sus problemas explica mejor que los grandes rasgos de la política nacional, el cuadro educativo. Durante el centralismo las políticas venian desde la capital hasta los departamentos, que vigilaban de cerca las actividades municipales.

Imaginar como uniformemente desolado este cuadro sería menospreciar las excepciones como Guadalajara, donde Manuel López Cotilla fundó varios establecimientos educativos. Morelia también se preocupaba por enseñar las primeras letras y Guanajuato en 1845 consideraba bien atendidas sus escuelas. Pero fuera de las zonas urbanas populosas, los municipios luchaban con la carencia de hombres capaces de encargarse del ramo de instrucción primaria. El país tenía un índice de analfabetismo de un $80 \%$, que en las comunidades rurales sin duda llegaba fácilmente al $90 \%$. El aislamiento de las comunidades dificultaba no solamente la llegada de libros, noticias y novedades, sino de maestros y de personas capaces de estimular la vida intelectual. La ayuda del clero era indispensable. En toda la República hubo un total de 3000 eclesiásticos, de los cuales apenas la mitad se dedicaba a la cura de almas y a tener un contacto estrecho con los creyentes. ${ }^{6}$ Este ejército potencial de maestros se vio disminuido por la edad, la guerra, la expulsión de los españoles, y no pudo reponerse por la falta de obispos, únicos que podrían ordenar nuevos sacerdotes. Había más municipios que parroquias o cabezas de doctrina, así que ni siquiera se contaba en teoría con un ministerio de culto en muchos de ellos.

La población de los municipios estaba dispersa en pequeñas rancherías sobre grandes extensiones de tierra, a veces de geografía muy accidentada. México, en el siglo pasado, tenía

\footnotetext{
da, La educación ilustrada (1786-1836). Educación primaria en la ciudad de México, México, El Colegio de México, 1977, $280 \mathrm{pp}$.

6 Anne Staples, La iglesia en la primera república federal mexicana: 1824-1835, México, Sepsétentas, 237.
} 
pocas ciudades. Se calcula que la capital tenia alrededor de un cuarto de millón de habitantes; Guadalajara, para los primeros años de independencia, no llegaba a $50000 .^{7}$. Abundaban en cambio pueblos y rancherías de 100 o 200 habitantes, donde ningún recurso alcanzaba para pagar un maestro.

Los municipios conseguían sus fondos principalmente de impuestos sobre el comercio. El derecho de plaza era su único recurso en muchas partes. Algunos lograron gravar los bailes o la introducción de pulque u otras bebidas embriagantes. Estas entradas eran esporádicas, impredecibles, y no permitían mantener una escuela sobre bases económicas firmes. Las escuelas que dependían del escaso interés y dinero de los municipios tuvieron una vida muy azarosa, con numerosas interrupciones. Con frecuencia faltaba el maestro ya que el escaso sueldo no alentaba a desempeñar esta tarea bajo condiciones tan penosas.

En Puebla, por ejemplo, el gobierno estatal asignaba los recursos locales de cada ayuntamiento. El de Tochimilco supo que el Congreso del estado, en 1831, le había dado "cuatro reales, tres, dos y uno semanarios sobre las tiendas y cantinas del país, calificadas en cuatro clases según su mayor o menor expendio; el de uno, dos y tres reales mensuales, bajo la misma clasificación, sobre las tabernas de pulque, y el de un octavo o tlaco sobre cada cuero de esta bebida que se introduzca de fuera para su venta". ${ }^{8}$ Le cobraba, pues, un impuesto graduado sobre tiendas y cantinas de aguardiente y licores nacionales y sobre tabernas de pulque, más una pequeña cantidad adicional sobre pulque introducido al municipio. Quedamos sin saber si lo recaudado de este impuesto alcanzaba para pagar decorosamente a un maestro.

Algunos ayuntamientos carecían de fondos, as tal grado que el estado los tenía que socorrer. El de Puebla en 1826 subvencionó una escuela gratuita en el barrio de San Antonio, mientras el ayuntamiento arreglaba sus finanzas. El estado des-

7 Viviane Bracht, La población de los estados mexicanos (1824-1895). México, Instituto Nacional de Antropología e Historia, 1976, (Colección Cientifica 35), p. 66.

\& Colección de acuerdos y decretos expedidos por el primer congreso constitucional ten sus últimas sesiones extraordinarias, y por el segundo y tercero en los años de 1830 y 1831, Puebla, Imprenta del supremo gobierno del estado, 1832, p. 98. 
tinaba 25 pesos mensuales para pagar al maestro y otra cantidad para cubrir la renta de la casa que servía de local. ${ }^{9}$

La escasez de dinero afectó de varias maneras a la educación antes de la República Restaurada. Evidentemente limitaba las opciones gubernamentales, pues el poco dinero recaudado se destinaba a la burocracia y al ejército. Prácticamente no hubo construcción de edificios públicos durante los años veinte y treinta, salvo el caso curioso de los cementerios, que si no requerían grandes caudales sí indicaban un interés de parte de las autoridades por sanear el ambiente. Durante un año tomado al azar, 1845, hubo doce solicitudes para construir cementerios, varias para cárceles y ninguna para escuelas. El poco dinero en caja se destinaba a arreglar y acondicionar cementerios y cárceles, después palacios municipales, puentes, caminos y cañería para agua potable y en último lugar las escuelas. Los edificios que servían de locales escolares eran todos construcciones coloniales; algunas dañadas seriamente durante la guerra de Independencia permanecían semidestruidas.

Los niños llegaban a compartir el mismo techo con compañeros poco deseables; en un pueblo, los presos ocupaban una parte del edificio, la escuela otra.

El distraer los fondos de instrucción pública para otros fines fue bastante común durante la primera parte del siglo. A veces lo hacía el gobierno estatal, al ordenar contribuciones forzosas de los ayuntamientos, que se veían obligados a tomar dinero de donde lo hubiera. A veces el mismo ayuntamiento ocupaba los fondos para la compra de maíz cuando no había suficiente, o para conseguir agua potable. Hubo necesidad de varias leyes para evitar que utilizaran los fondos educativos con otros propósitos.

La Compañía Lancasteriana, bajo el centralismo de Santa Anna, fue la primera organización que puso algún remedio a estas dificultades ya que logró, por lo menos sobre el papel, dos cosas: uniformar y centralizar la educación en toda la República, darle un solo reglamento y un solo método, y extender este sistema a todos los departamentos obligatoriamente.

9 Colección de los decretos y órdenes más importantes que expidió el primer congreso constitucional del estado de Puebla en los años 1826, 1827 y 1828, Puebla, Imprenta del gobierno, 1828 , p. 82. 
Logró también dotar al sistema de fondos, cobrados a razón de un real por mes a cada jefe de familia, salvo en casos de notoria pobreza. ${ }^{10}$ En la práctica, duró poco la Compañía como directora de educación primaria, y durante su breve actuación no cumplió con sus propias metas. Pero dejó un poderoso precedente y en algunas partes se formaron posteriormente juntas de instrucción pública que continuaron los métodos impuestos por la Compañía. Desgraciadamente, la práctica de cobrar a los padres de familia que podían pagar no se volvió a instituir hasta el imperio de Maximiliano, gobierno que como sabemos tampoco se consolidó.

Fue muy criticada por los opositores del régimen centralista la actuación de la Compañía Lancasteriana, en parte porque no entregó sus estados financieros. El entonces ministro José María Lafragua criticaba esta falta de seriedad y el hecho de que en el Distrito Federal, por ejemplo, quedaban para 1847 apenas tres escuelas para niñas en condiciones deplorables, sin fondos suficientes para pagar a sus maestras. Sin ser culpa de la Compañía, Lafragua señalaba a las autoridades como apáticas, incapaces de proteger debidamente a la instrucción pública y vigilar el adecuado cumplimiento de la ley. "Los esfuerzos", decía el ministro, "han sido inútiles". No había logrado vencer la rutina, introducir novedades, ni conseguir mayores medios pecuniarios. ${ }^{11}$

Tampoco llevaba bien sus cuentas el gobierno. Un informe de 1861 indica que la Junta de Instrucción Pública no había entregado cuentas en 14 años. Establecido en Guanajuato en 1858, el gobierno de Juárez trató de mejorar las condiciones

10 Manuel Dublán y José María Lozano, La legislación mexicana o colección completa de las disposiciones legislativas expedidas desde la independencia de la república, México, Imprenta del Congreso, 1876-1904, IV, p. 311 . El noveno artículo de la ley de 26 octubre 1842 dice que si no alcanzaban los fondos para establecer por lo menos una escuela de niños y otra de ninas por cada 10000 habitantes, se cobraría una "pensión de un real, que pagarán mensualmente los cabezas de familia, tenga o no hijos, exceptuándose solamente los que sean notoriamente pobres". Para evitar este impuesto muy impopular los departamentos se apresuraron a enviar informes favorables sobre los recursos disponibles para sus escuelas.

"Memoria de la primera secretaría de Estado y del despacho de Relaciones Exteriores de los Estados Unidos Mexicanos leida al Supremo Congreso Constitucional por el ministro del ramo C. José María Lafragua en diciembre 1846. México, Imprenta de Vicente García Torres, 1847, pp. 104, 105. 
educativas pero la falta de fondos y la mala voluntad de los reaccionarios, según el decir de los liberales, arruinó los establecimientos educativos. Los conservadores cerraron algunos y derrocharon los fondos de otros, escribia Manuel Ruiz, ministro de Negocios Eclesiásticos e Instrucción Pública en $1861 .^{12}$

Se comprende que la organización de escuelas haya sido dificil en la provincia, dadas las características de los ayuntamientos. Sorprende, en cambio, encontrar situaciones desastrosas tan cerca de la capital, como era la prefectura y comandancia militar de Hidalgo, compuesta por lo que es ahora San Juan de Aragón, Zacatenco y Atzcapotzalco. Al revisar la situación de esta zona en abril de 1867 el prefecto no encontró una sola escuela, ni el recuerdo siquiera de alguna ley que reglamentara su existencia y que le procurara fondos. Luchando contra "la rancia costumbre e inercia de los pueblos", establecieron el mes siguiente varias escuelas para niños y niñas, tarea que, por la brevedad del tiempo transcurrido, parece imposible. Si no son exactos los datos, de todas maneras nos dan una idea del tamaño de las escuelas y de los sueldos de los maestros. Había dos municipios en esta prefectura; en uno se establecieron siete escuelas a las que asistian 565 niños. Los sueldos de los maestros fluctuaban entre 12 y 55 pesos mensuales, cantidades proporcionales a la asistencia de estudiantes. El otro municipio tenía cinco escuelas, 517 alumnos y el sueldo de los maestros era de 15 a 30 pesos. Cada municipio tenía una escuela para niñas; a una asistían 110 niñas, cuya maestra ganaba 30 pesos al mes; otra tenía 30 niñas y la maestra ganaba 20 pesos. Como la mayoría de las escuelas de esta época, un solo maestro enseñaba simultáneamente todos los niveles.

En el otro extremo del valle, en la municipalidad de Tlalpan, los ocho maestros con que se contaba ganaban entre $15 \mathrm{y}$ 52 pesos mensuales. El de Topilejo, poblado más lejano, ganaba menos que el de San Pedro Mártir, a pesar de tener más alumnos. San Angel tenía seis maestros, con sueldos muy parecidos, Coyoacán otros tantos, Ixtapalapa e Ixtacalco, regiones netamente indígenas, cinco cada uno. Xochimilco, que aparece

12 Exposición que el C. Lic. Manuel F. Ruiz, ministro que fue de Justicia, ministro de Negocios Eclesiásticos e Instrucción Pública, presentó al Supremo Congreso..., México, Imprenta de Nicolás Pizarro, 1861, p. 43. 
en las estadísticas de la época como prefectura política y ciudad, tenía un total de 33 escuelas para niños y tres para niñas, que pagaban sueldos de 8 a 40 pesos mensuales. Las tres preceptoras ganaban exactamente la mitad de lo asignado a sus compañeros del sexo opuesto. En la cabecera de Xochimilco, por ejemplo, el maestro ganaba 40 pesos y la maestra 20. En Milpa Alta se repetía la situación y uno de sus pueblos, que tenía escuela de niñas, destinaba 10 pesos a la maestra y el doble al maestro. Las escuelas se dividían en tres clases, según lo completo de su enseñanza, y esto afectaba también el nivel de los sueldos.

La ciudad de México en 1867 tenía ocho escuelas bajo la dirección de la Compañía Lancasteriana, que si ya no estaba encargada de la instrucción pública en toda la República, no por eso había dejado de existir. El municipio sostenía a diez escuelitas, cuya población apenas llegaba a la mitad de las ocho lancasterianas. Había en la ciudad 123 escuelas particulares donde se pagaba algún tipo de colegiatura. ${ }^{13}$ En total, apenas cubrían malamente las necesidades educativas de unos mil alumnos, cantidad ínfima para una ciudad de más de un cuarto de millón de habitantes. Ejemplos como éstos se repartían a lo largo y ancho del territorio nacional.

Al investigar la creación de institutos de ciencias y artes en varios lugares de la República y el esfuerzo del gobierno por mantener a los colegios nacionales, nos inclinamos a pensar que realmente sí tuvieron un respaldo económico importante. Estos establecimientos pertenecían al ramo de-enseñanza superior, no a la primaria. Daban mayor prestigio al gobierno y por ello acaparaban su interés. Las escuelas de primeras letras, en contraste, fueron el patito feo, el renglón olvidado de la administración pública. Los particulares trataron de organizarlos en las ciudades como México y en las haciendas, pero la mayor parte de los municipios tenian que resolver el problema con sus propios recursos.

Algunos ejemplos pueden ilustrar este punto. En 1838 el ministro del Interior admitió que los fondos de los ayunta-

${ }^{19}$ Memoria que el secretario de estado y del despacho de Justicia e Instrucción Pública presenta al Congreso de la Unión en marzo de 1868, México, Imprenta del gobierno en palacio, 1868, pp. 93-98, cuadro 4. 
mientos todavía estaban "muy desarreglados", pero que bajo el régimen central las juntas departamentales quedarían ahora encargadas de establecer escuelas en todos los pueblos y de dotarlos de fondos propios e imponer contribuciones moderadas a los habitantes. ${ }^{14}$ Pero no se resolvía así el problema ya que los ayuntamientos tendrían que conseguir fondos de su propia localidad mediante impuestos, recurso poco efectivo si no había excedentes de ningún tipo.

Para la década de los años cuarenta, Manuel Baranda, joven abogado de Guanajuato, era ministro de Justicia y Negocios Eclesiásticos. Según sus cálculos exageradamente optimistas, habría unas cinco mil escuelas en la República con un cuarto de millón de alumnos, poquísimos para un territorio tan amplio como era entonces el mexicano, con unos ocho millones de habitantes. Según un informe preparado por la oficina de Baranda, las muchas escuelas de primeras letras que existían antes de la Independencia se habían multiplicado hasta el infinito con lo que México se encontraba en el nivel de los países más civilizados y avanzados del orbe. En el mismo informe se dice que "en nuestras montañas del norte, entre los precipicios y barrancas, lugares que parecen inhabitables, ha existido una escuela tan completa y tan perfectamente sistematizado que haría honor a la capital ". ${ }^{15}$ Nadie más observó semejante perfección ni romanticismo en las escuelas elementales mexicanas de esos años, al contrario, todos lamentaban sus deficiencias.

La República Restaurada, en una época de mayor tranquilidad política, tuvo con su labor resultados más positivos. Los fondos destinados a la instrucción pública se obtuvieron mediante partidas presupuestarias constantes, aunque el pago oportuno de sueldos siguió siendo problemático por la desviación de fondos. La incomunicación subsistía en gran medida y los maestros apenas empezaban a gozar de cierta respetabili-

\footnotetext{
14 Memoria del ministerio de lo Interior de la república mexicana leida en las cámaras de su congreso general en el mes de enero, 1838, México, Imprenta del Águila, 1838, p. 3.

15 "Memoria que en forma de dictamen presentó la comisión permanente, a la junta general de instrucción pública sobre este ramo, en cumplimiento de la ley de 11 de agosto 1843 y pasada al gobierno por acuerdo de la misma junta". Publicado en $E l$ Monitor Republicano y reproducido en El Siglo XIX, 28-31 agosto 1845.
} 
dad social. Se venció la inercia y por fin se estableció definitivamente la costumbre de tributar algún tipo de impuesto para sostener la instrucción pública. La penuria tan marcada de los primeros años cedió a la disponibilidad de recursos, aunque limitados, que permitieron, junto con el esfuerzo del gobierno, maestros y padres de familia, dar origen al sistema de educación nacional. 